\title{
The Calyx of Held Develops Adult-Like Dynamics and Reliability by Hearing Onset in the Mouse In Vivo
}

\author{
Mandy Sonntag, ${ }^{1 \star}$ Bernhard Englitz, ${ }^{1,2,3 \star}$ Marei Typlt, ${ }^{1}$ and Rudolf Rübsamen ${ }^{1}$ \\ ${ }^{1}$ Faculty of Biosciences, Pharmacy and Psychology, University of Leipzig, 04103 Leipzig, Germany, ${ }^{2}$ Max-Planck-Institute for Mathematics in the Sciences, \\ 04103 Leipzig, Germany, and ${ }^{3}$ Neural Systems Laboratory, University of Maryland, College Park, Maryland 20742
}

The development of the auditory system has received increasing attention since the mechanisms of patterned, spontaneous activity in prehearing mammals were discovered. This early activity originates in the cochlea and is assumed to be of importance for the establishment and refinement of synaptic connections in the auditory system. In the present study we investigate synaptic transmission and its interplay with spontaneous discharges in the developing auditory system. We used the calyx of Held as a model system, where this question can be investigated in vivo over a broad range of ages [postnatal day 8 (P8)-P28]. To precisely quantify the timing and reliability of synaptic transmission, we developed a novel fitting approach which decomposes the extracellularly recorded signal into its presynaptic and postsynaptic components. In prehearing mice, we found signal transmission to be unreliable, with high variability in the transmission delay and in the amplitude of postsynaptic components. These timing and amplitude changes were strongly correlated with the preceding activity. Around hearing onset (P12-P14), the properties of signal transmission converged to the adult-like state which was characterized by high transmission reliability as well as high consistency in timing and amplitude. Although activity-dependent depression was still found in action potentials, EPSP depression no longer played a prominent role. In conclusion, the maturation of synaptic transmission at the calyx of Held seems to be precisely timed to achieve its adult potential by the time acoustically evoked signal processing commences.

\section{Introduction}

The development of the mammalian auditory system has attracted increased interest since the mechanisms of cochleainduced activity before the onset of acoustically evoked activity were discovered (Tritsch et al., 2007). In the prehearing phase, auditory nerve fibers (ANFs) receive excitatory input originating in the cochlea, although this activity is not sound induced. Instead, ATP is released transiently in the cochlea, which depolarizes the hair cells, leading to bursts of spikes in ANFs. This activity is assumed to influence the structure, strength, and function of synaptic connections in the developing auditory system (Friauf and Lohmann, 1999; Rubel and Fritzsch, 2002; Erazo-Fischer et al., 2007; McKay and Oleskevich, 2007). To study synaptic transmission in the developing auditory system in detail, the calyx of Held in the medial nucleus of the trapezoid body (MNTB) is a well suited model system because of its exceptional accessibility.

\footnotetext{
Received Feb. 2, 2011; revised March 18, 2011; accepted March 21, 2011.

Author contributions: M.S., B.E., and R.R. designed research; M.S. and B.E. performed research; M.S., B.E., M.T., and R.R. contributed unpublished reagents/analytic tools; M.S. and B.E. analyzed data; M.S., B.E., M.T., and R.R. wrote the paper.

Financial support was provided by the Deutsch Forschungsgemeinschaft (DFG) (Ru 390/18-2 and Ru 390/19-1). B.E. was supported partly by a DFG Forschungsstipendium (EN 919/1-1) and M.S. and M.T. by the DFG graduate college InterNeuro (GRK 1097). We thank Timm Lochmann, Rainer Stollhoff, Stephan Poppe, and Neil Rabinowitz for interesting discussions on the fitting procedure, and two anonymous reviewers for valuable comments on the manuscript.

*M.S. and B.E. contributed equally to this work.

Correspondence should be addressed to Rudolf Rübsamen, Institute of Biology II, University of Leipzig, Talstrasse 33,04103 Leipzig, Germany. E-mail: rueb@uni-leipzig.de.

DOI:10.1523/JNEUROSCI.0575-11.2011

Copyright $\odot 2011$ the authors $\quad 0270-6474 / 11 / 316699-11 \$ 15.00 / 0$
}

A number of in vitro studies have investigated synaptic transmission at the developing calyx of Held (Taschenberger and von Gersdorff, 2000; Futai et al., 2001; Joshi and Wang, 2002), even without the knowledge of the temporal structure of spontaneous activity and its developmental changes (Sonntag et al., 2009; Tritsch et al., 2010). The ambient activity can, however, be an important determinant for the properties of synaptic transmission, as shown in a recent study (Hermann et al., 2007).

In the present study, we therefore investigated the development of synaptic transmission in vivo under the naturally occurring activity patterns. We performed extracellular recordings at the calyx of Held-MNTB principal cell junction, which allowed the same recording and analytical techniques to be applied across a range of ages from postnatal day 8 (P8) to P28 with the neural system kept intact on both the network and the cellular level. To study the precise timing and amplitudes of the presynaptic and postsynaptic action potential (AP), as well as the EPSP, we developed a novel fitting approach which allowed a precise decomposition of individual transmission events into these components. Signal transmission could then be characterized in terms of speed and reliability, and of stability of postsynaptic signal amplitude.

Before the onset of hearing, we found signal transmission to be unreliable and of pronounced variability in transmission delay as well as in amplitude of the EPSP and the postsynaptic AP. The preceding activity could be shown to strongly predict this timing and amplitude variability. Around hearing onset (P12-P14), signal transmission attained the properties found at the mature calyx of Held, i.e., reliable in AP transmission with low variability in both the transmission delay and the amplitude of the postsynap- 
A

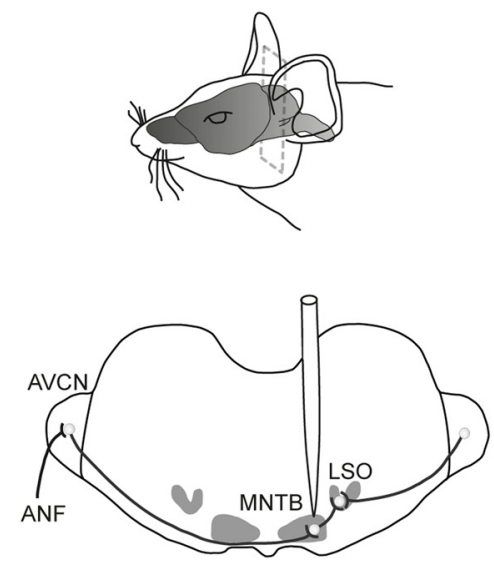

Extracellular

voltage recordings

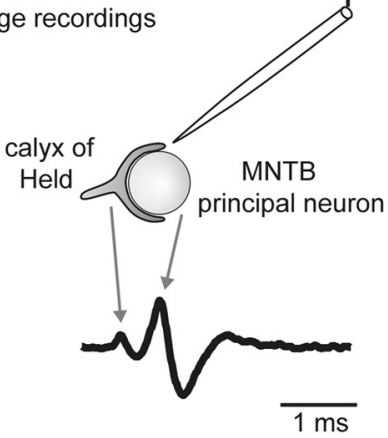

B

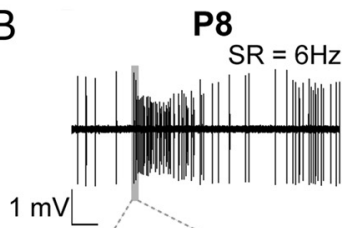

$1 \mathrm{~s}$

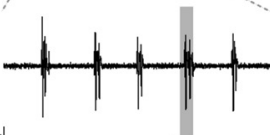

$1 \mathrm{mV}$

$50 \mathrm{~ms}$

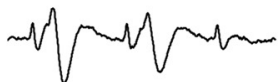

$1 \mathrm{mV}$

$1 \mathrm{~ms}$

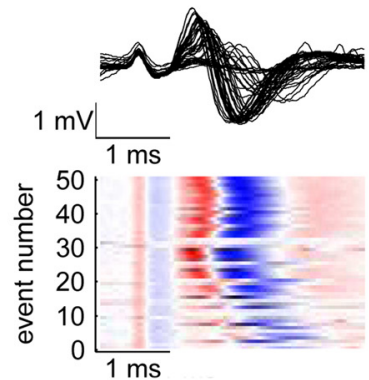

C

P11 $\mathrm{SR}=26 \mathrm{~Hz}$

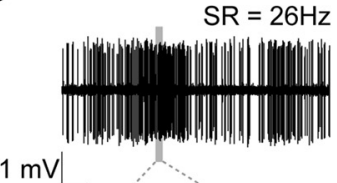

$1 \mathrm{~s}$

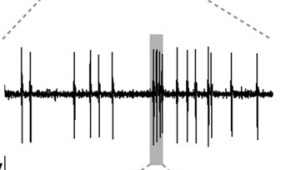

$1 \mathrm{mV}$

$50 \mathrm{~ms}$

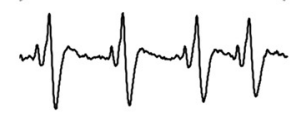

$1 \mathrm{mVL}$

$1 \mathrm{~ms}$

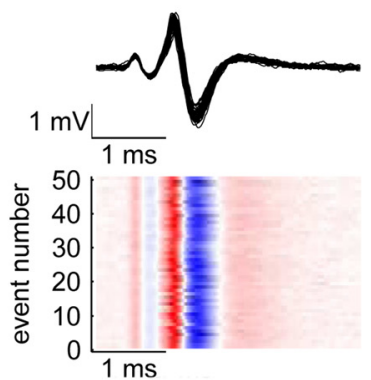

P28
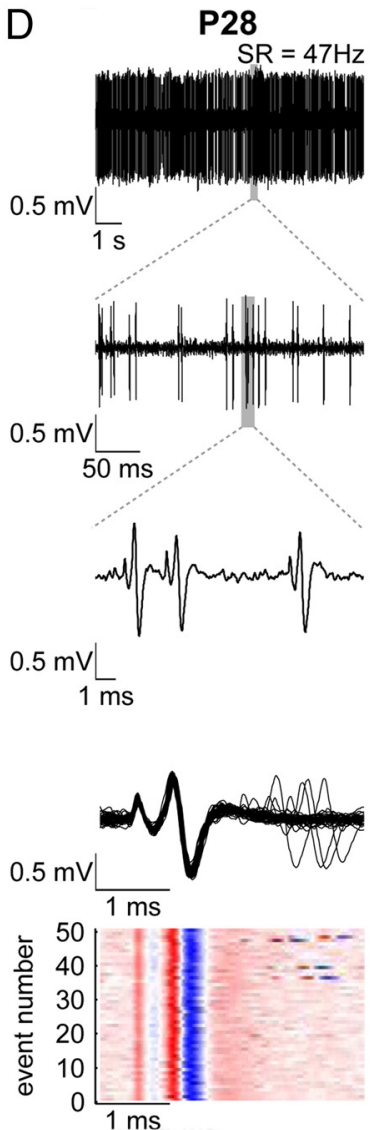

Figure 1. Overview of the recording setup and patterns of discharge activity at different postnatal ages. A, An in vivo preparation for extracellular single-unit recordings. Top and middle, The MNTB was approached by a glass electrode penetrating from the dorsal side through the cerebellum and the brainstem. Bottom, Recordings yielded complex voltage signals consisting of the calyx AP followed by the postsynaptic potential. $\boldsymbol{B}-\boldsymbol{D}$, Patterns of spontaneous activity at three different ages. The spontaneous rates are indicated in the top row. $\boldsymbol{B}$, At P8, the spontaneous activity is characterized by discrete bursts of transmission events interrupted by silent or low-activity periods (first row). Within single bursts, the patterned activity is structured further displaying series of $1-5$ transmission events in close succession, i.e., mini-bursts (second and third row). Within these mini-bursts complex waveforms exhibited high variability in amplitude and temporal relations of presynaptic and postsynaptic components (third row). The fourth row shows 50 overlaid complex potentials triggered to the presynaptic AP, and the fifth row visualizes in a color plot the variability of the different signal components on the same time scale. The red color indicates the size of the positive phases and the blue color of the negative phases of the complex signal. Shown here is a series of spontaneous discharge activity in its natural occurring succession. The light-colored horizontal stripes indicate transmission events, in which the presynaptic signal component is followed by strongly reduced postsynaptic components. C(P11), D (P28), At older ages the discharge activity becomes more continuous even though bursting discharges can be still present at P11. The complex waveforms shorten and attain their adult-typical, constant shape. AVCN, Anteroventral cochlear nucleus; LSO, lateral superior olive; SR, spontaneous rate.

tic AP. While AP depression was still observed at high levels of preceding activity, no evidence for short-term depression (STD) was found (Lorteije et al., 2009). In summary, we found signal transmission at the calyx of Held to mature in time for the onset of acoustically evoked signal processing.

\section{Materials and Methods}

\section{Animals and preparation}

All experiments were approved by the Saxonian District Government, Leipzig. Data were collected from CBA/J mice ( 7 males and 6 females) and C57BL/6J mice ( 3 males and 6 females) at P8 ( $n=7$ single-units), $\mathrm{P} 10(n=6), \mathrm{P} 11(n=8), \mathrm{P} 12(n=6), \mathrm{P} 14(n=5), \mathrm{P} 23(n=5)$, and P28 $(n=5)$. Data from both mouse strains were pooled here, since both in the present and in a previous comparative study (Sonntag et al., 2009) no substantial differences were found between the strains with respect to the investigated properties. The animals were initially anesthetized by an intraperitoneal injection of a mixture of ketamine hydrochloride $(0.1$ $\mathrm{mg} / \mathrm{g}$ body weight, Pfizer) and xylazine hydrochloride $(0.005 \mathrm{mg} / \mathrm{g}$ body weight, Bayer). Anesthesia was maintained during the recording session by supplementary injections of one-third of the initial dose when necessary. A small metal bolt was glued on the prefrontal skull to hold the animal in a stereotaxic recording device. For insertion of recording electrode and reference electrode, two holes $(\varnothing 0.5 \mathrm{~mm})$ were drilled at a distance of $1.8-2 \mathrm{~mm}$ caudal to the lambda suture at the midline and 1-1.5 mm lateral to the midline, respectively. For verification of recording site, Fluoro-Gold (FG) was iontophoretically injected ( $4 \mu \mathrm{A}, 10 \mathrm{~min}$ ) after the electrophysiological experiments. The animal was perfused 5-24 $\mathrm{h}$ after FG injection with $0.9 \%$ sodium chloride and $4 \%$ paraformaldehyde before isolating the brain. The brain was cut with a vibratome (50 $\mu \mathrm{m}$ slices) and the recording sites were visualized under the fluorescence microscope.

\section{Data collection}

The experiments were performed in a sound-attenuated chamber (type 400, Industrial Acoustics Company $\mathrm{GmbH}$ ) on a vibration-isolated table. The animals were positioned on a temperature-controlled heating pad keeping the body temperature at $\sim 38^{\circ} \mathrm{C}$. We exclusively performed extracellular single-unit recordings using high-impedance glass micropipettes with filament (5-12 M $\Omega$, GB150F-10, Science Products) filled with $3 \mathrm{M} \mathrm{KCl}$. The MNTB was approached dorsally and reached at penetration depths of 5-6 mm. Recordings in the proximity of the calyx of Held yielded a complex voltage waveform, which reflects a single transmission event and is composed of the presynaptic AP in the calyceal terminal, and the activity of the postsynaptic MNTB principal cell constituted by an EPSP and (typically) the postsynaptic AP (Fig. 1A) (see also Guinan and Li, 1990; Kopp-Scheinpflug et al., 2003; Lorteije et al., 2009). The recorded voltage signals were amplified (Neuroprobe 1600, A-M Systems; 
A1
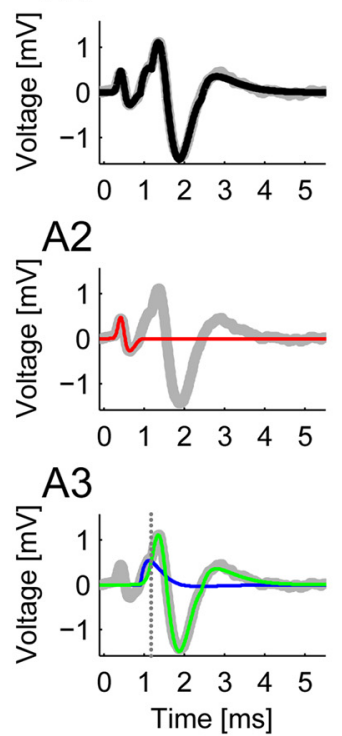

B

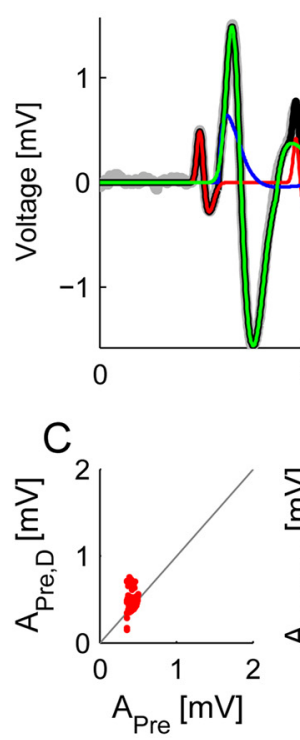

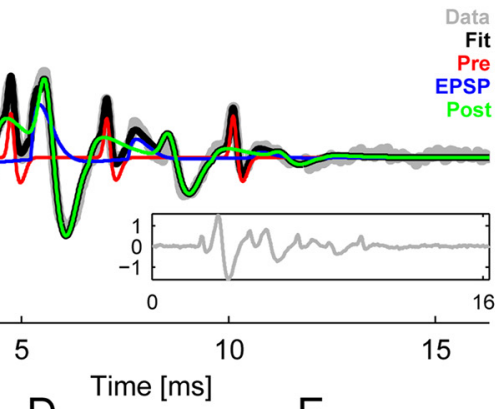
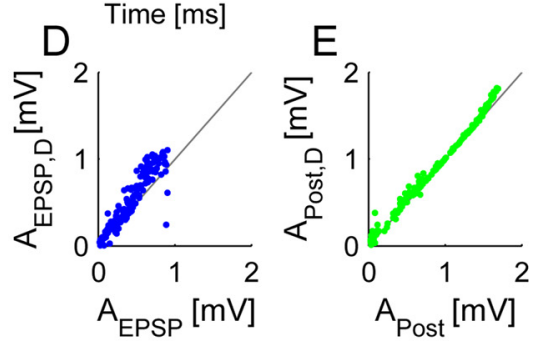

Figure 2. Precise quantification of the complex waveforms variability requires a new fitting procedure. $\boldsymbol{A}, \mathrm{A}$ typical complex waveform (gray) is composed of the presynaptic AP and a postsynaptic component consisting of the EPSP and the postsynaptic AP. A parameterized sum of the above components provided an excellent fit (black) to the complex waveform. The components were modeled using a biphasic kernel for the presynaptic $\operatorname{AP}(\boldsymbol{A} \mathbf{2}$, red), a biphasic kernel for the $\operatorname{EPSP}(\boldsymbol{A} \mathbf{3}$, blue) and a triphasic kernel for the postsynaptic AP $(\boldsymbol{A} \mathbf{3}$, green). The vertical, dotted line marks the time when the AP replaces the EPSP in the overall fit. $\boldsymbol{B}$, In a mini-burst the complex waveforms overlap and exhibit variability in size and temporal spacing. We used a sum of complex waveform fitting functions to account for these issue, e.g., in the depicted mini-burst 4 complex waveforms occurred where in the last one the EPSP was rather slow leading to a failure in eliciting a postsynaptic AP (original data in gray; see also inset). The sum (black) closely traces the voltage trace, while the individual components (colors as in $\boldsymbol{A}$ ) are estimated corrected for the overlap, e.g., the size of the presynaptic AP is corrected for the coincident presence of the postsynaptic AP's third phase. $\boldsymbol{C}-\boldsymbol{E}$, The sizes of the different components estimated via the fit correspond to the direct measurement from the voltage trace, except when correcting overlaps. C, Especially for the presynaptic AP this correction is necessary, since its size is often overestimated when events occur close to each other (group of dots above the diagonal). $\boldsymbol{D}$, The correspondence between the fitted EPSP size and the inflection point in the postsynaptic waveform is not as reliable, since for large APs the inflection point diminishes and becomes hard to measure. $\boldsymbol{E}$, For the AP the correspondence is usually quite good with small noise-induced positive deviation of the measurement (since the maximum over a certain range is computed). The average fit accuracy was $99.6 \%$, measured in explained variance corrected for noise (see Results for details).

PC1, Tucker-Davis Technologies), bandpass-filtered $(0.3-7 \mathrm{kHz})$, and digitized by an A/D converter (RP2.1, Tucker-Davis Technologies) at a sampling rate of $97.7 \mathrm{kHz}$.

For each single-unit, spontaneous activity was recorded for 5-10 min. In animals between P12 and P28, we additionally recorded the neuronal activity under acoustical stimulation to verify age-matched hearing capacity (see Sonntag et al., 2009, for further information on the stimulus protocols).

\section{Acoustic stimulation}

The acoustic stimuli were generated by custom-written MATLAB software (MathWorks). Following D/A conversion (RP2.1, Tucker-Davis Technologies), the signals were presented via electrostatic couplers (ES1, Tucker-Davis Technologies) controlled by an electrostatic speaker driver (ED1, Tucker-Davis Technologies). The acoustic stimuli were delivered through a plastic tube ( $\varnothing 5 \mathrm{~mm}$ ) tagged to the electrostatic coupler and ending just in front of the eardrum.

\section{Data selection}

Single-units were only included in the analysis if at least 400 transmission events were collected during recording of spontaneous activity (on average $741 \pm 185$ transmission events/unit; range, [404,1160], $n=42$ ), and the signal-to-noise ratios of the presynaptic $\mathrm{AP}\left(\mathrm{SNR}_{\mathrm{Pre}}: 9.3 \pm 5.7\right.$, range, $[3.6,30.4], n=42)$ and postsynaptic $\mathrm{AP}\left(\mathrm{SNR}_{\text {Post }}: 19.8 \pm 8.3\right.$, range, $[7.9,42.5], n=42$ ) exceeded 3 (computed as the average positive peak divided by the SD of the noise).

\section{Data analysis}

Quantification of transmission events. The transmission events in the extracellular recordings exhibited substantial variation in relative timing and size of the subcomponents (Fig. $1 B)$, i.e., the presynaptic AP, and the postsynaptic EPSP and AP. Quantification of these variations, especially when consecutive transmission events partially overlap in time, requires decomposing the transmission event into these three subcomponents. This task is unfortunately outside the scope of classical spike-sorting algorithms, where the waveforms are assumed to not overlap and remain constant in shape [an exception to the first assumption was presented by Herbst et al. (2008), still requiring substantial computational resources]. Therefore, a dedicated fitting procedure was developed in MATLAB (MathWorks). In short, each transmission event or sequence of transmission events was represented as a weighted sum of presynaptic and postsynaptic potentials, which could exhibit a well controlled amount of shape and size changes (Fig. 2).

More precisely, the $k$ th transmission event $W_{\mathrm{TE}, k}\left(t ; p_{k}\right)$ (Fig. 2A1) was represented as a sum of potentials emitted from the presynaptic calyx $W_{\text {Calyx }, k}\left(t ; p_{k}\right)$ (Fig. $\left.2 A 2\right)$ and the postsynaptic MNTB principal cell $W_{\mathrm{PC}, k}\left(t ; p_{k}\right)$ (Fig. $2 A 3)$, reflecting the linear summation of electromagnetic fields in the extracellular medium, i.e., $W_{\mathrm{TE}, k}\left(t ; p_{k}\right)=W_{\mathrm{Calyx}, k}\left(t ; p_{k}\right)+W_{\mathrm{PC}, k}\left(t ; p_{k}\right)$. The index $k$ denotes the position of a given event in the whole sequence of transmission events, and $p_{k}$ denotes the set of parameters of the $k$ th transmission event. Each potential is a basic function of time $t$, parameterized by $p_{k}$. In the following, $p_{k}$ is written for simplicity, although each subcomponent only uses a subset of the parameters in $p_{k}\left(W_{\text {Calyx }, k}: 6, W_{\mathrm{PC}, k}: 6\right.$ for the EPSP, 9 for the postsynaptic AP).

The calyx potential was modeled as a basic, bipolar waveform $W_{\text {Pre }}\left(t ; p_{k}\right)$ (a sum of two Gaussian bell curves) scaled by a parameter $A_{\text {Pre, } k}$, i.e., $W_{\text {Calyx; } k}\left(t ; p_{k}\right)=$ $A_{\text {Pre }, k} W_{\text {Pre }}\left(t ; p_{k}\right)$. This shape reflects the extracellular potential corresponding to an intracellular spike, obtained approximately as a linear combination of the intracellular voltage and its derivative (Lorteije et al., 2009).

Similarly, the postsynaptic part was modeled as a sequence of the EPSP and an action potential, $W_{\mathrm{PC}, k}\left(t ; p_{k}\right)=\left(1-H_{t_{k}}\right) A_{\mathrm{EPSP}, k} W_{\mathrm{EPSP}, k}\left(t ; p_{k}\right)+$ $H_{t_{k}} A_{\text {Post }, k} W_{\text {Post }, k}\left(t ; p_{k}\right)$ (Fig. $2 A 3$, blue and green curves, respectively), where the transition between the two happens at the time point $t_{\mathrm{th}}$, here indicated by a heavyside step-function $H_{t_{k}}$ (Fig. $2 \mathrm{A3}$, dotted vertical line). No additivity was assumed here between EPSP and AP for two reasons. First, the two events are not additive, since the elicitation of the AP will change the driving potential for the synaptic conductance underlying the EPSP. Second, the size of the postsynaptic AP (or rather its slope) will be determined by the total conductivity, and our estimates thus accurately reflect this property of the AP. As for the calyx potential, $W_{\mathrm{EPSP}}\left(t ; p_{k}\right)$ and $W_{\text {Post }}\left(t ; p_{k}\right)$ were both modeled as basic waveforms, the former bipolar (sum of two $\alpha$ functions) and the latter tripolar (sum of two Gaussian bell curves followed by an $\alpha$ function). Each of the basic waveforms $W_{\text {Pre }}(t$; $\left.p_{k}\right), W_{\text {EPSP }}\left(t ; p_{k}\right)$, and $W_{\text {Post }}\left(t ; p_{k}\right)$ was automatically adapted in shape to the waveforms in each recording, reflected by the parameters $p_{k}$. These parameters included the temporal location of the waveform, the peak widths, and relative heights. The basic waveforms were each normalized to their positive peak amplitude, such that the parameters $A_{\mathrm{Pre}, k}, A_{\mathrm{EPSP}, k}$, and $A_{\text {Post }, k}$ represent the actual, positive amplitudes of each component. 
If multiple waveforms were close in time $(<5 \mathrm{~ms}$ between their presynaptic APs) and overlaps became likely, they were treated together as a sequence of transmission events $S_{\mathrm{TE}, i}(t)$, modeled as a sum of multiple waveforms $W_{\mathrm{TE}, k}\left(t ; p_{k}\right)$, i.e., $S_{\mathrm{TE}, i}(t)=W_{\mathrm{TE}, k}\left(t ; p_{k}\right)+W_{\mathrm{TE}, k+1}\left(t ; p_{k+1}\right)$ $+\ldots$, where the length of the sequence was increased until the improvement stopped outweighing the increase in the number of parameters. This balance was assessed by the Bayesian information criterion (Schwarz, 1978).

In total, 21 parameters defined a transmission event. Fitting all 21 parameters would have become unfeasible especially for sequences of transmission events. Hence, 7 parameters were chosen based on their biological importance and/or large range of variability. These were the amplitudes $A_{\mathrm{Pre}, k}, A_{\mathrm{EPSP}, k}$, and $A_{\mathrm{Post}, k}$, the time of the presynaptic peak, the delay from the presynaptic peak to the EPSP peak, and the delay from the EPSP peak to the postsynaptic peak, as well as the relative size of the postsynaptic peaks. The remaining 14 parameters were initially adapted for the temporally separated transmission events in a given recording and subsequently set to their average in the final fitting run on all transmission events in a given recording. Using simulations and assessing the distributions of the 14 parameters for the separated events, it was verified that these parameters did not exhibit substantial, systematic variations.

At its core, the fitting process ran a nonlinear fit, based on the MATLAB function, lsqnonlin. Because of the substantial number of parameters when fitting mini-bursts $(7 \times$ number of events, i.e., up to 50$)$ and multiple restarts to escape local minima, fitting a single mini-burst could take hours. Human supervision was used to speed up this process by suggesting the position of the presynaptic APs. Importantly, these user suggestions did not constrain the fit, as the resulting fit was only accepted if its residual was close to the noise level. Further, human supervision guaranteed a quality control for each fitted event.

The accuracy of the fitting method was tested by generating surrogate data with matched statistics for a selection of recordings and reestimating the varied parameters, which provided highly correlated agreement $(r=0.82-1)$ between estimates and true values. Fitting accuracy was quantified in terms of the fraction of explained variance, i.e., $f_{\mathrm{VE}}=\left\langle\frac{\operatorname{Var}\left(S_{i}\right)-\operatorname{Var}\left(S_{i}-\hat{S}_{i}\right)}{\operatorname{Var}\left(S_{i}\right)}\right\rangle$, and the fraction of explained variance corrected for the variance contributed by voltage noise, i.e., $f_{\mathrm{VE}}=\left\langle\frac{\operatorname{Var}\left(S_{i}\right)-\operatorname{Var}\left(S_{i}-\hat{S}_{i}\right)}{\operatorname{Var}\left(S_{i}\right)-\operatorname{Var}_{\text {noise }}}\right\rangle$, where $S_{i}$ is the data, $\hat{S}_{i}$ the corresponding fit of one or more waveforms, and $V r_{\text {noise }}$ the variance of the noise (estimated from non-spiking sections).

Failures of AP transmission. A general concern for extracellular recordings is the possibility of obtaining a mixture of signals from multiple cells. In an earlier study (Englitz et al., 2009), we developed a statistical criterion for deciding whether the recordings stem from one or multiple sources, the Independence Assessment of Potentials (IAP). IAP was routinely computed for each recording after extracting and subtracting the fits to the postsynaptic potentials fitted above. If a recording violated the IAP criterion, and thus was likely to contain signals from more than one calyx-principal cell junction, it was excluded from further analysis.

If a recording was confirmed to be reliably single-unit, individual failures of AP transmission were identified on the basis of their fitted amplitude $A_{\text {Post }}$. In the presence of AP failures, one expects the histogram of $A_{\text {Post }}$ to become bimodal, with one peak around the typical amplitude of the AP and another peak at noise level (for an example, see Fig. $3 F$ ). Note that $A_{\text {Post }}$ will always be slightly positive, since it was limited by 0 from below in the fitting procedure and will be fit to small noise fluctuations in the absence of an actual AP (Fig. $2 B$, green curve). If such a second peak was present in $<15 \%$ of the maximum $A_{\text {Post }}$ (on average, $7.3 \%$, range $[2,12] \%)$, we searched for the minimum between the peaks and classified all transmission events below this value as failures of AP transmission.

Measures of previous activity. The properties of spiking have been shown to depend on previous activity (Fedchyshyn and Wang, 2007; Mc Laughlin et al., 2008; Lorteije et al., 2009; Tolnai et al., 2009). Usually, the last interspike interval (ISI) is used as the independent variable. Since previous activity extending beyond one preceding ISI can be influential (Tolnai et al., 2009), we additionally used a weighted average of all pre- ceding transmission events, weighted by the distance in time and amplitude of the respective events. This quantity was computed for both the presynaptic and the postsynaptic AP train, termed calyx-activity (see Fig. $5 A 2$ ) and principal cell (PC)-activity (see Fig. 5A3), respectively. Especially in juvenile animals, the PC-activity can differ strongly from the calyx-activity, e.g., in cases where the postsynaptic AP is strongly reduced in its amplitude and/or delayed with respect to the presynaptic AP.

The weighting was implemented as an exponentially decaying kernel, emphasizing close transmission events over far transmission events, i.e., for an AP at $t_{0}$ the previous activity was computed as $\operatorname{Act}\left(t_{0}\right)=\frac{1}{\operatorname{median}\left(A_{i}\right)} \sum_{i=-1}^{-\infty} A_{i} e^{\left(t_{i}-t_{0}\right) / \tau}$. Here, the $A_{i}$ values are either $A_{\text {Pre }}$ or $A_{\text {Post }}$ for the calyx- or PC-activity, respectively. Division by the median amplitude renders the calyx- and PC-activity directly comparable and improves the comparability between different recordings. We used a time constant, $\tau=100 \mathrm{~ms}$, while others (30-200 ms) gave quite similar results. Since the results from these activity measures lead to a linearization over a wide range (see Fig. 5), we consider it a better approximation to activation state of the synapse.

Statistical analysis. Data are provided as mean \pm SD in Figure 3 to illustrate the variability and mean $\pm 2^{\star}$ SEM below in Figures 5 and 6 , where group comparisons are made. For comparisons across age involving multiple age groups, standard ANOVA tests were used to detect changes as a function of age. Correlations between two quantities were assessed by Spearman rank correlation to cover linear and nonlinear relationships.

\section{Results}

We quantified the properties of synaptic transmission in 42 calyx of Held/MNTB principal cell recordings in vivo at seven postnatal ages between P8 and P28. We exclusively used extracellular recordings, which enables simultaneous acquisition of the activity of the calyx of Held and the corresponding MNTB principal cell reflected in a complex waveform (Fig. $1 A$ ). The range of postnatal ages covers the early stages before the onset of hearing (P8-P11, prehearing), the period of onset and maturation of acoustically evoked signal processing (P12-P14), and the mature hearing condition (P14-P28). We focused on signal transmission during spontaneous activity to allow a comparison between prehearing and hearing stages, but occasionally also analyzed sound-driven activity.

\section{Spontaneous activity matures from patterned to sustained discharges}

Before hearing onset (P8-P10), the spontaneous activity appears in bursts of discharges interrupted by periods of reduced activity or silence (Fig. $1 B$, first row). Typically, single bursts are composed of small groups of 1-5 transmission events (i.e., miniburst), which occur in relatively stereotypic temporal sequences (Fig. $1 B$, second row) (see also Tritsch et al., 2010). Particularly within these mini-bursts, we found a great variation in the amplitude of the postsynaptic AP and in the temporal relation of the presynaptic and postsynaptic components (Fig. $1 B$, third to fifth row). Also, transmission events lacking the postsynaptic AP were observed frequently before hearing onset (Fig. $1 B$, third to fifth row). The average spontaneous rates were low and similar for all units $(<25 \mathrm{~Hz})$ at $\mathrm{P} 8-\mathrm{P} 10$, while a high variability in spontaneous rates was found in units of older animals $(<1 \mathrm{~Hz}$ to $>100 \mathrm{~Hz})$ (see also Sonntag et al., 2009). Furthermore, bursting discharges vanished around hearing onset (P11-P12) and gradually developed into a more regular and continuous discharge activity (Fig. $1 C, D$, first and second row) (see also Sonntag et al., 2009). Along with the developmental changes in spontaneous activity pattern around hearing onset, we found less variation in amplitude and 
A

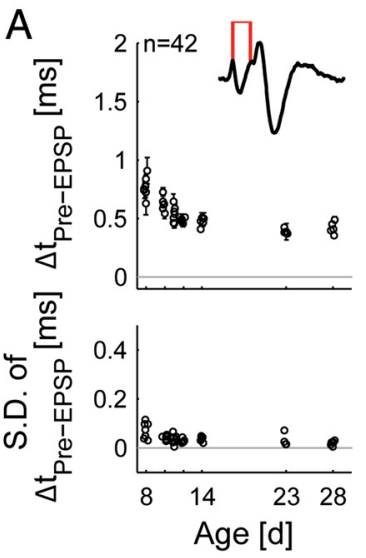

B
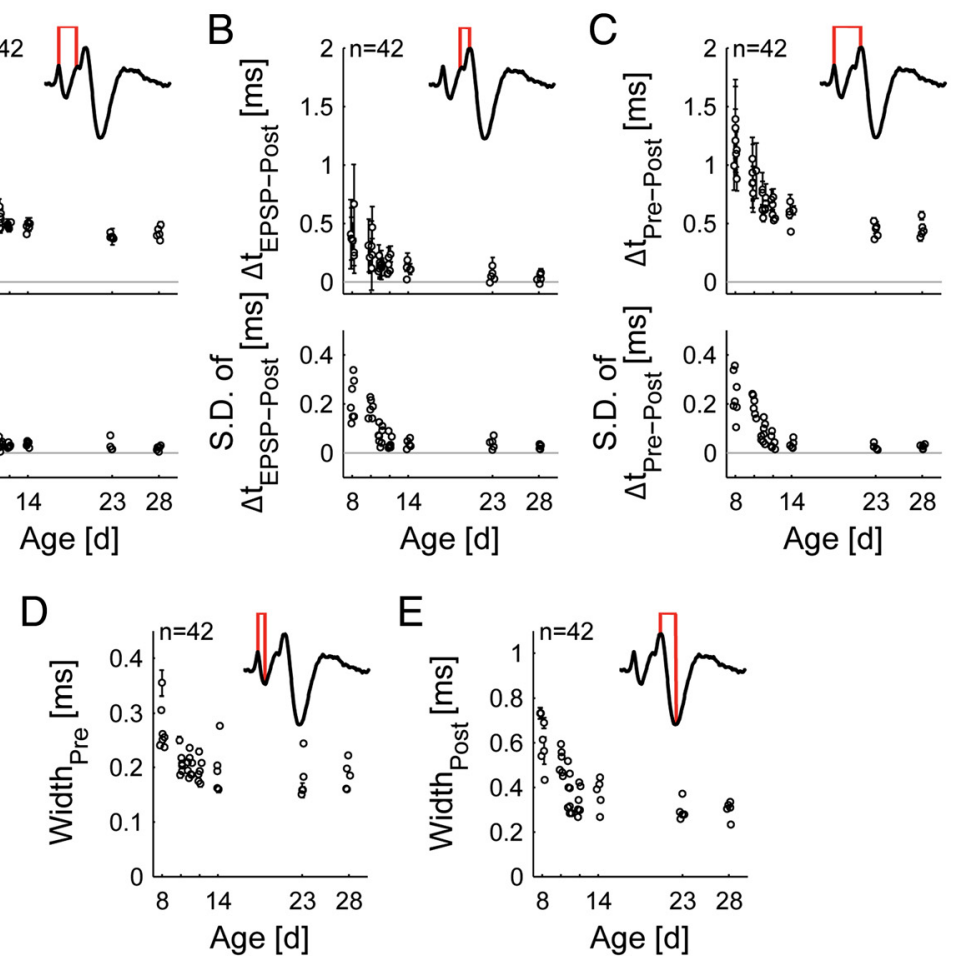

E
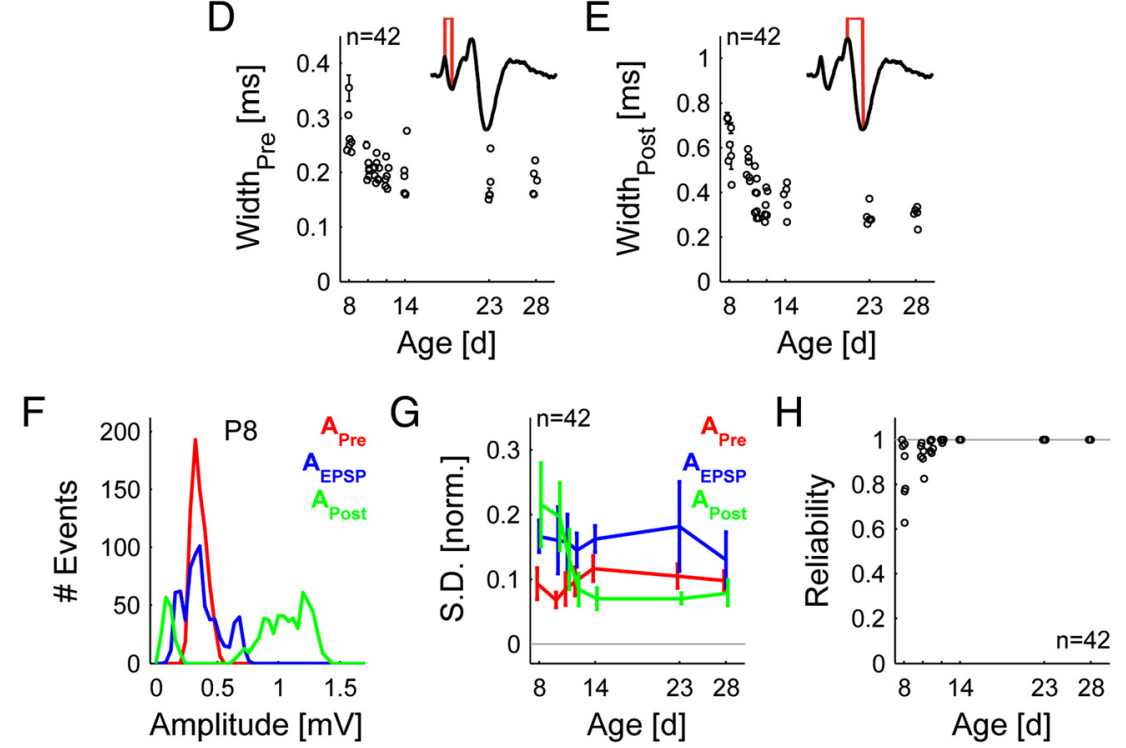

Figure 3. Dynamics and reliability of transmission events change during maturation reaching stable levels around hearing onset. $\boldsymbol{A}-\boldsymbol{C}$, The transmission delay speeds up (top) and becomes less variable (bottom) during maturation, both for the part from the peak of the presynaptic AP to the peak of the EPSP $(A)(p<0.001)$ and that from the peak of the EPSP to the peak of the postsynaptic $\mathrm{AP}(\boldsymbol{B})(p \ll 0.001)$, and also for the AP transmission delay $(\boldsymbol{C})$ (presynaptic AP peak to postsynaptic AP peak, $p \ll$ $0.001)$. $\boldsymbol{D}, \boldsymbol{E}$, The width of both the presynaptic $(\boldsymbol{D})$ and the postsynaptic AP $(\boldsymbol{E})$ decreased with age $(p \ll 0.001) \boldsymbol{F}$, The distribution of amplitudes of the three components differs in prehearing animals. The presynaptic AP amplitude $\left(A_{P r e}\right)$ is tightly distributed, while the EPSP $\left(A_{\text {EPSP }}\right)$ and the postsynaptic AP $\left(A_{\text {Post }}\right)$ amplitudes show much wider distributions. If AP transmission fails, the distribution of $A_{\text {Post }}$ becomes bimodal (as in the P8 example depicted here). Due to the presence of noise and the EPSP, the fitted $A_{\text {post }}$ amplitudes are positive rather than $0 . G$, These results were further quantified by the SD normalized by dividing by the maximal value for each variable. Only $A_{\text {Post }}$ shows a significant change as a function of age $(p \ll 0.001)$, while $A_{\text {Pre }}$ and $A_{\text {EPSP }}$ did not systematically change across age. $\boldsymbol{H}$, Reliability of transmission increases significantly with age, reaching practically flawless transmission around hearing onset. Data are plotted as mean $\pm S D$ and were obtained from 42 single-units with $5-8$ units per age.

in timing of the different components of the transmission events (Fig. 1C,D, third to fifth row).

\section{New fitting approach captures the properties of single transmission events}

The variability in timing and amplitude within a transmission event poses a challenge to classical spike sorting procedures, which assume constant waveshapes. Further, the frequent occurrence of overlaps-both between adjacent transmission events and within a given transmission event-complicates the precise measurement of the amplitudes of each subcomponent. We developed a novel fitting approach whose design explicitly incorporates these challenges (see Materials and Methods for details). Each transmission event is decomposed into its constituent presynaptic and postsynaptic components (Fig. 2A1-3), i.e., the pre- synaptic AP (red), the EPSP (blue), and the postsynaptic AP (green). Accordingly, sequences of transmission events are decomposed into sequences of the respective presynaptic and postsynaptic components (Fig. 2B). Overlaps between the components are considered in two different ways. First, presynaptic and postsynaptic components are summed, as is done in the extracellular medium. Second, the EPSP is assumed to be replaced at some point in time by the postsynaptic AP (corresponding to the time of $\mathrm{AP}$ initiation; Fig. 2A3, dotted line). The shape of each component was constrained by only a few parameters, most of which were estimated for a given recording, while only seven parameters characterized the amplitudes and internal timing of one transmission event (see Materials and Methods).

This approach allowed measuring the size of each component even within overlaps and is more resistant to noise than conventional approaches. This is demonstrated in Figure $2 C-E$ for the presynaptic AP (red), the EPSP (blue), and the postsynaptic AP (green). Each plot shows the fitted values (abscissa) compared to values measured directly from the raw data (i.e., estimated as the absolute maximum in the vicinity of the fitted maximum for the presynaptic and postsynaptic APs and as the inflection point for the EPSP). First, the noise resistance can be seen for individual transmission events in Figure $2 B$, but also in Figure $2 C-E$, where the directly measured values fall slightly above the diagonal. This is a consequence of brief noise fluctuations that are being picked up by the criterion of taking a (local) maximum as the amplitude. Second, the resistance to overlaps is exemplified in the second and third presynaptic AP (red) in Figure $2 B$ and is also the source of the predominance of points (even a cluster) above the diagonal in Figure 2C. The measurement of the EPSP's amplitude is generally more complicated if the EPSP and the postsynaptic AP are elicited very close in time. As shown in Figure $2 D$, the measurements are consistent with the measurement of the inflection point (Lorteije et al., 2009), which generally exhibits similar variability under these circumstances.

Overall, the present method accurately represented each transmission event as indicated by the average explained variance of $97.3 \%$ (corresponding approximately to $r=0.99$ ). When accounting for the variance of the voltage noise (assumed to be independent), the explained variance rises to $99.6 \%$. Fitting quality was quite constant across ages.

\section{Signal transmission dynamics mature up to hearing onset}

We first analyzed the development of transmission speed in vivo. We estimated the timing of two main steps of AP transmission: (1) the delay between the presynaptic AP's positive peak and the 
Table 1. Developmental changes in transmission speed

\begin{tabular}{llrrr}
\hline Parameter & P8 $(n=7)$ & P14 $(n=5)$ & P28 $(n=5)$ & \multicolumn{1}{l}{$\begin{array}{l}\text { Significance } \\
\text { (ANOVA) }\end{array}$} \\
\hline$\Delta t_{\text {Pre-Post }}$ & $1.15 \pm 0.17 \mathrm{~ms}$ & $0.58 \pm 0.09 \mathrm{~ms}$ & $0.45 \pm 0.07 \mathrm{~ms}$ & $p<<0.001$ \\
$\Delta t_{\text {Pre-EPSP }}$ & $0.77 \pm 0.09 \mathrm{~ms}$ & $0.47 \pm 0.04 \mathrm{~ms}$ & $0.42 \pm 0.05 \mathrm{~ms}$ & $p<<0.001$ \\
$\Delta t_{\text {EPSP-Post }}$ & $0.38 \pm 0.05 \mathrm{~ms}$ & $0.1 \pm 0.04 \mathrm{~ms}$ & $0.03 \pm 0.04 \mathrm{~ms}$ & $p<<0.001$ \\
SD of $\Delta t_{\text {Pre-Post }}$ & $0.24 \pm 0.09 \mathrm{~ms}$ & $0.03 \pm 0.02 \mathrm{~ms}$ & $0.03 \pm 0.01 \mathrm{~ms}$ & $p<<0.001$ \\
SD of $\Delta t_{\text {Pre-EPSP }}$ & $0.07 \pm 0.01 \mathrm{~ms}$ & $0.04 \pm 0.01 \mathrm{~ms}$ & $0.02 \pm 0.01 \mathrm{~ms}$ & $p<0.001$ \\
SD of $\Delta t_{\text {EPSP-Post }}$ & $0.21 \pm 0.08 \mathrm{~ms}$ & $0.04 \pm 0.02 \mathrm{~ms}$ & $0.02 \pm 0.01 \mathrm{~ms}$ & $p<<0.001$ \\
Width $_{\text {Pre }}$ & $0.27 \pm 0.04 \mathrm{~ms}$ & $0.2 \pm 0.04 \mathrm{~ms}$ & $0.19 \pm 0.03 \mathrm{~ms}$ & $p<<0.001$ \\
Width $_{\text {Post }}$ & $0.61 \pm 0.11 \mathrm{~ms}$ & $0.37 \pm 0.07 \mathrm{~ms}$ & $0.3 \pm 0.04 \mathrm{~ms}$ & $p<<0.001$ \\
\hline
\end{tabular}

Speed of signal transmission was calculated as the time between (1) the positive peak of the presynaptic AP and the peak of the EPSP $\left(\Delta t_{\text {Pre-EPSP }}\right)$, (2) the peak of the EPSP and the positive peak of the postsynaptic AP ( $\left.\Delta t_{\text {EPSP-Post }}\right)$, and (3) the sum of $\Delta t_{\text {Pre-EPSP }}$ and $\Delta t_{\text {EPSP-Post }}\left(\Delta t_{\text {Pre-Post }}\right)$. The dynamics of the presynaptic and postsynaptic AP were measured as the time between the positive and negative peak (Width Pre $_{\text {end }}$ Width Post $_{\text {. }}$ ).

EPSP's peak ( $\Delta t_{\text {Pre-EPSP }}$, Fig. $\left.3 A\right)$, closely related to the classical synaptic delay, and (2) the delay between the EPSP's peak and the postsynaptic AP's positive peak $\left(\Delta t_{\text {EPSP-Post }}\right.$, Fig. $\left.3 B\right)$. The sum of these two delays constitutes the AP transmission delay $\left(\Delta t_{\text {Pre-Post }}\right.$, Fig. 3C) (Tolnai et al., 2009).

Consistent with observations in brainstem slices (Taschenberger and von Gersdorff, 2000), we found a developmental decrease in $\Delta t_{\text {Pre-Post }}$ (Fig. $3 C$, top, $p \ll 0.001$ ) from P8 to P14 when AP transmission almost achieved adult-like speed (Table 1). This developmental increase in speed derives from a shortening of $\Delta t_{\text {Pre-EPSP }}$ (Fig. $3 A$, top, $p \ll 0.001$; Table 1 ) and $\Delta t_{\text {EPSP-Post }}$ (Fig. $3 B$, top, $p \ll 0.001$; Table 1$)$.

We further found a prominent age-dependent decline in the jitter of transmission speed (equal to SD, Fig. $3 A-C$, bottom; also see decrease in error bars in Fig. $3 A-C$, top). In a total of 7 units at P8, $\Delta t_{\text {Pre-Post }}$ of individual AP transmission events varied from $0.41 \mathrm{~ms}$ to $3.26 \mathrm{~ms}$ (mean jitter: $0.24 \pm 0.09 \mathrm{~ms}$ ). Across age, the jitter in $\Delta t_{\text {Pre-Post }}$ was greatly reduced (Table 1 ). The high variability in $\Delta t_{\text {Pre-Post }}$ at prehearing stages was mostly attributed to the high variability in $\Delta t_{\text {EPSP-Post }}$ (e.g., P8: $0.21 \pm 0.08 \mathrm{~ms}[n=$ $7]$ ), since the jitter in $\Delta t_{\text {Pre-EPSP }}$ was found to be quite low (e.g., P8: $0.07 \pm 0.01 \mathrm{~ms},[n=7]$ ) (Table 1$)$. In summary, the synaptic delay (i.e., $\Delta t_{\text {Pre-EPSP }}$ ) was longer in the early second postnatal week compared to older ages, but the synaptic transmission was nonetheless quite stable at these young stages. However, the timing of postsynaptic AP generation after the EPSP's onset was found to be highly variable before hearing onset.

The speedup of signal transmission goes along with a shortening of presynaptic and postsynaptic APs from P8 to P14 with only slight reductions in the further development (Fig. $3 D, E, p$ $\ll 0.001$; Table 1). Considering the changes of presynaptic and postsynaptic APs, also developmental changes in the kinetics of the EPSP are to be expected. However, the EPSP time constants could only reliably be determined at younger ages where EPSPs were visible in cases of AP transmission failures. Thus, we are not able to make clear assumptions about the development of the EPSP time course and have therefore omitted the data here.

\section{Postsynaptic AP size and transmission reliability stabilize during development}

Next, we analyzed developmental changes in the amplitudes of the presynaptic and postsynaptic potentials which serve as a measure for neuronal excitability. Before hearing onset, the relative size of the presynaptic AP $\left(A_{\text {Pre }}\right)$ was stable throughout a recording, reflected by a narrow amplitude distribution (for an example of a P8 mouse, see Fig. $3 F$, red), while the amplitude of the EPSP $\left(A_{\mathrm{EPSP}}\right)$ and, even more so, the postsynaptic AP $\left(A_{\text {Post }}\right)$ showed wide distributions (Fig. 3F, green and blue), indicating a high variability in amplitude within a single recording. In the case of AP failures, the distribution of the AP even becomes bimodal with a second peak at very low amplitudes (Fig. $3 F$, green).

To quantify and compare the variability in signal size between different ages, we estimated the normalized SD of $A_{\text {Pre }}, A_{\text {EPSP }}$, and $A_{\text {Post }}$, termed relative variability (normalized by dividing by the maximal value for each component). The relative variability of $A_{\text {Pre }}$ was generally low and did not systematically change throughout development (Fig. $3 G$, red). The relative variability of $A_{\text {EPSP }}$ was approximately twofold larger than the one of $A_{\text {Pre }}$ but, likewise, no significant developmental changes were found (Fig. $3 G$, blue). In contrast, the relative variability of $A_{\text {Post }}$ was significantly larger before the onset of hearing, attaining the mature state around $\mathrm{P} 12$, when the relative variability of $A_{\text {Post }}$ was in approximately the same range as for $A_{\text {Pre }}$ (Fig. $3 H$, green; $p \ll$ $0.001)$.

In the present dataset, failures of AP transmission, i.e., lack of postsynaptic APs following EPSPs, were present from P8 to P12 (Fig. $3 H$ ). At P8, AP failures were found in 5 of 6 units, with failure rates varying between $2 \%$ and $37 \%$. In the further development, both the number of units exhibiting AP failures and the failure rates decreased (P12; AP failures in 2 of 5 units, failure rates $0.2 \%$ and $1.4 \%$ ) up to $\mathrm{P} 14$, when no AP failures were found anymore. These results are in accordance with Mc Laughlin et al. (2008) and Englitz et al. (2009), but not with Lorteije et al. (2009). Note that $A_{\text {Post }}$ 's age-dependent decrease in relative variability (Fig. $3 G$, green) is partly due to the changes in transmission reliability.

\section{Variability in transmission delay covaries with variability in amplitude}

In the preceding sections, we demonstrated that in prehearing stages, both the postsynaptic signal amplitudes $\left(A_{\mathrm{EPSP}}\right.$ and $\left.A_{\text {Post }}\right)$ and the AP transmission delay exhibited substantial variability. Next, we checked for correlations between these two observations. While we found $A_{\text {Pre }}$ to be independent of the following transmission delay at all postnatal ages (P8 example, Fig. 4A1; P28 example, Fig. $4 \mathrm{B1}$; all units, Fig. $4 C 1$; different colors indicate age: from red [P8] to blue [P28]), $A_{\mathrm{EPSP}}$ and $A_{\text {Post }}$ were strongly correlated with the AP transmission delay before hearing onset (Fig. 4A2,3). Furthermore, we found that failures of AP transmission typically went along with small EPSP amplitudes (Fig. 4A4, gray dots). In older animals, a smaller range of AP transmission delays is covered, and only slight correlation with $A_{\mathrm{EPSP}}$ and $A_{\text {Post }}$ is found (P28 example, Fig. 4B2,3, enlargement of the data in the inset; all units: Fig. 4C2,3). We also found a positive correlation between $A_{\mathrm{EPSP}}$ and $A_{\text {Post }}$ in prehearing animals, while with increasing age this correlation is weakened or even absent (P8 example, Fig. 4A4; P28 example, Fig. 4B4; all units, Fig. 4C4).

\section{Preceding activity predicts variations in postsynaptic AP amplitude and transmission delay}

Recent in vivo studies in young adult mice $(\sim$ P32) and adult cats reported an activity-dependent decrease of $A_{\text {Post }}$ at very short ISIs (Mc Laughlin et al., 2008: AP depression for ISI < 20 ms; Lorteije et al., 2009: AP depression for ISI $<10 \mathrm{~ms}$ ). We found a similar effect, which was strongly pronounced during early postnatal development (consistent with the Fig. $3 G$ ). Across all ages $A_{\text {Post }}$ exhibited a nonlinear relationship with respect to the ISI $_{\text {Pre }}$ (between presynaptic APs), i.e., a reduction in $A_{\text {Post }}$ for shorter ISI $_{\text {Pre }}$ which leveled off for longer ISI $_{\text {Pre }}$. Both the magnitude in ampli- 

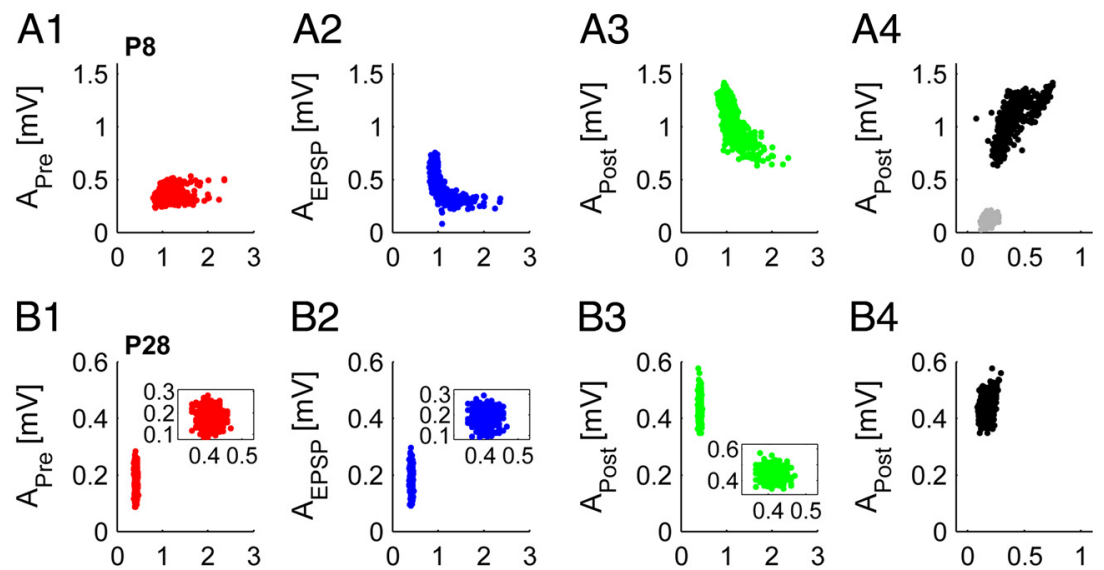

B2

B3

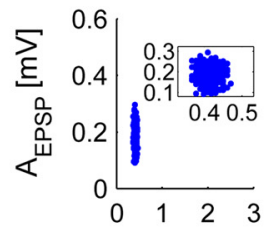

C1

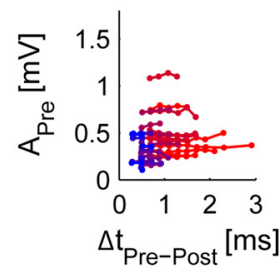

$\mathrm{C} 2$
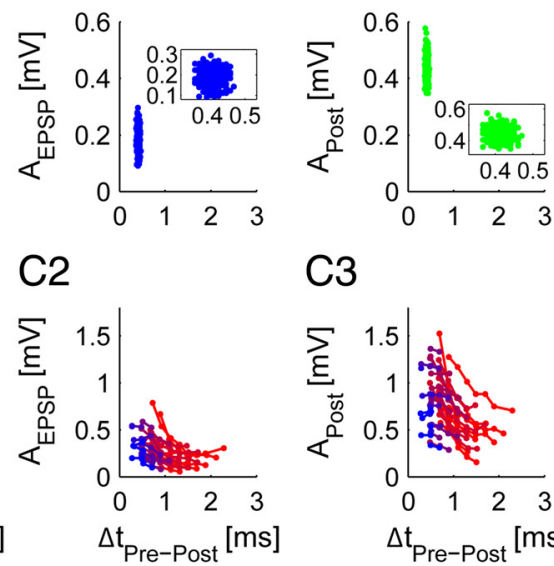

B4

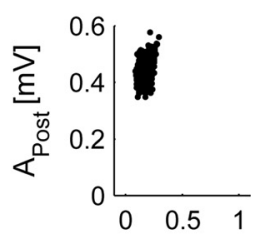

C3

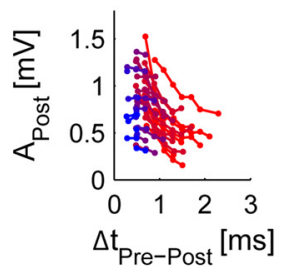

C4

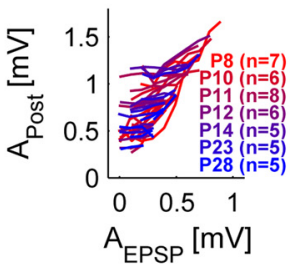

Figure 4. Transmission delay and postsynaptic amplitudes covary in the early development. $A$, In an example unit of a prehearing animal (P8) the presynaptic AP amplitude $\left(A_{\text {Pre }}\right)$ was independent of the following transmission delay (A1), while $A_{\text {EPSP }}(\boldsymbol{A} \mathbf{2})$ and $A_{\text {Post }}(\boldsymbol{A} \boldsymbol{3})$ decreased with increasing delays. Also, $A_{\text {EPSP }}$ and $A_{\text {Post }}$ were well correlated (A4). Transmission events lacking the postsynaptic AP had small EPSP amplitudes (A4, gray dots). $\boldsymbol{B}$, In an example unit of a P28 animal, $A_{\text {Pre }}(\boldsymbol{B} 1), A_{\text {EPSP }}$ (B2), and $A_{\text {Post }}$ (B3) were not related to the transmission delay, and $A_{\text {EPSP }}$ did not depend on $A_{\text {Post }}(B 4)$. $\boldsymbol{C}$, Correlations of all 42 single-units (different colors indicate different ages: from red [P8] to blue [P28]; see also legend in (4 for unit numbers). At all postnatal ages, $A_{\text {Pre }}$ was independent of the following transmission delays $(\mathbf{C} 1) . A_{\text {EPsp }}$ and $A_{\text {Post }}$ were well correlated to the transmission delays in prehearing animals while in later development some correlation remained, however, covering only a small range of transmission delays $(\mathbf{C}, \mathbf{C})$. Also, $A_{\text {EPSP }}$ systematically increased with increasing $A_{\text {Post }}$ in prehearing animals, while in older animals $A_{\text {EPSP }}$ became independent of $A_{\text {post }}$ (C4). Binning was chosen uniform for all cells. Only bins with 5 or more transmission events were evaluated. Note that in $\mathbf{C 4}$, markers were left away for better visualization.

tude reduction and the ISI $\mathrm{Pre}_{\mathrm{Pr}}$ where the falloff started decreased with age (Fig. 5).

Examples of spontaneous activity for different ages are shown in Figure 5B1 (P8), C1 (P11), and D1 (P23). Successful transmissions are depicted in black, failures of AP transmission in gray. For the two prehearing examples the falloff is clearly visible and highly significant (P8, $r=0.81, p \ll 0.001$, Fig. 5B1; P11, $r=$ $0.46, p \ll 0.001$, Fig. $5 C 1$ ). The P23 example shows that-in some cases- the spontaneous rates were not high enough to sample the $\operatorname{ISI}_{\text {Pre }}$ range in which the falloff occurs (Fig. 5D1). Using acoustic stimulation for the same unit, the firing rates could be elicited in the range where the falloff in $A_{\text {Post }}$ occurs, albeit only to a lesser degree than at younger ages (for ISI Pre $_{2}<4 \mathrm{~ms}, r=0.39, p$ $\ll 0.001$, Fig. 5E1). On the population level, the age dependence in both amplitude and ISI $_{\mathrm{Pre}}$ falloff point was retained (Fig. 5F1, different colors indicate age: from red [P8] to blue [P28]), with the ISI ${ }_{\text {Pre }}$ falloff point moved to $\sim 3-4 \mathrm{~ms}$ for hearing animals (P14-P28). The population data only contain the successful transmission events, since the integration of AP failures would have biased the results for young animals. As is apparent from the example units, AP failures occurred dominantly at short ISI $_{\text {Pre }}$. As shown in Figure 4, the transmission delay correlates with $A_{\text {Post }}$, and consistently, the transmission delay also exhibits an activity dependence (Fig. 5G1). Note that this dependence was often nonmonotonic at $\mathrm{P} 8-\mathrm{P} 10$, in contrast to the monotonic dependence of $A_{\text {Post }}$. With maturation this dependence diminished, becoming almost flat at P28. Since the firing rates in the present study were comparably low, the submillisecond changes in transmission delay (Tolnai et al., 2009) are not easily discernible here, especially as a function of ISI $_{\text {Pre }}$ (see also below).

In addition to the ISI $_{\text {Pre }}$, we quantified the preceding activity by two more general measures, the calyx-activity (Fig. 5, middle column) and the PC-activity (Fig. 5, right column). While the ISI $_{\text {Pre }}$ measure just refers to the distance in time to the last AP transmission event, the calyx- and PCactivity correspond to the (exponentially) weighted activity of all preceding AP transmission events, embodying the notion that the activity level at the synapse depends on multiple preceding events weighted by the time since they occurred (Fig. 5A1-3; for details see Materials and Methods). The weighting with the AP amplitudes is intended to capture the level of activity for a single event. In juvenile animals, the PC-activity can differ strongly from the calyx-activity, e.g., if the postsynaptic AP is strongly reduced and delayed in the late phase of a mini-burst.

The correlations between $A_{\text {Post }}$ and $\Delta t_{\text {Pre-Post }}$ with both calyx- and PC-activity were comparable to the values from the ISI $_{\text {Pre }}$, with the main difference being a larger degree of linearization for individual units for the former two (Fig. 5F2$3, \mathrm{G} 2-3)$. This effect is largest for $\Delta t_{\text {Pre-Post }}$, where a non-monotonic (nonlinear) dependence becomes monotonic (linear). Especially in mini-bursts, the second transmission event usually has a very low ISI $_{\text {Pre }}$, yet its transmission delay is increased less than following transmission events in the mini-burst, which are preceded by a number of events (with comparable $\mathrm{ISI}_{\text {Pre }}$ ). A linear relationship usually indicates that the relationship between two quantities has been aptly modeled with only few remaining degrees of freedom left.

\section{Variation in EPSP amplitude is predicted by preceding activity in prehearing animals}

As mentioned above, we measured a high variation in the EPSP amplitude at all postnatal ages (Fig. 3G, blue). Thus, we checked whether the EPSP amplitude was dependent on the preceding activity (providing evidence for STD) and whether developmental differences were present. We found activity-dependent EPSP depression in prehearing but not in hearing animals (Fig. 6). In prehearing animals, the EPSP amplitude was dependent on ISI $_{\text {Pre }}$, and on the calyx- and PC-activity, respectively (example unit of a P8 mouse [ISI Pre $, r=0.72, p \ll 0.001$; calyx-activity: $r=-0.88$, $p \ll 0.001$; PC-activity: $r=-0.86, p \ll 0.001]$, Fig. 6A1-3; population data, Fig. 6C1-3; different colors indicate age: from red [P8] to blue [P28]). In older animals $A_{\mathrm{EPSP}}$ and the preceding activity are uncorrelated (example unit of a P28 mouse [ISI Pre, $r=-0.059, p=0.096$; calyx-activity, $r=-0.055, p=0.12$; PC-activity, $r=-0.053, p=0.13$ ], Fig. 6 B1-3; population data, Fig. 6C1-3). These data suggest that STD is present at immature calyces of Held, but does not play a role at the mature calyx of Held in vivo (Lorteije et al., 2009; Borst, 2010). In addition to 
A1
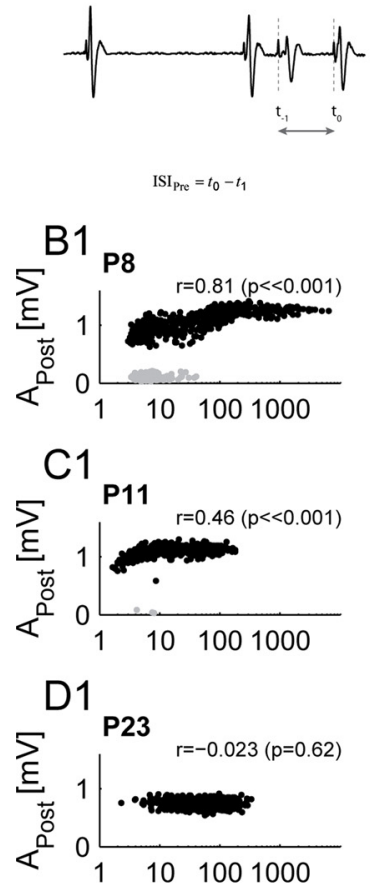

E1

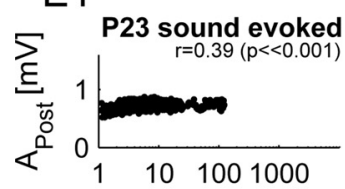

F1

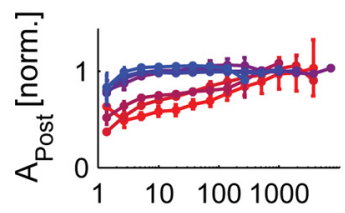

G1

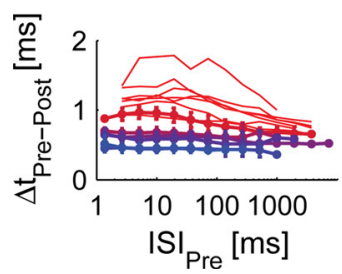

A2

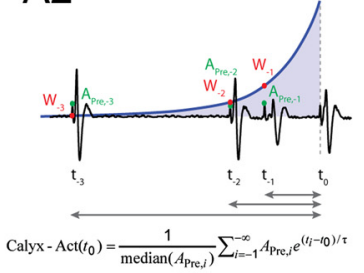

B2

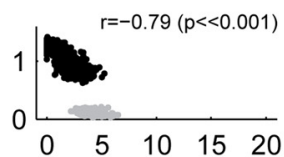

C2

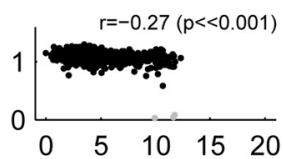

D2

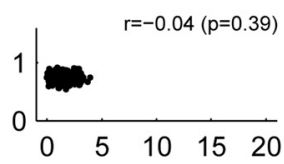

E2

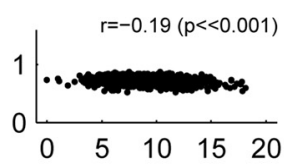

F2

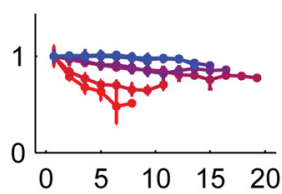

G2

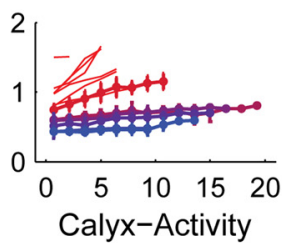

A3

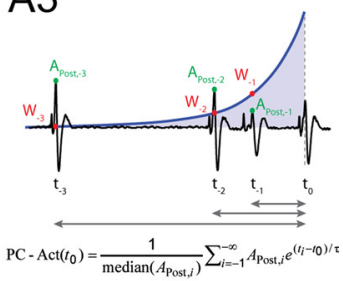

B3

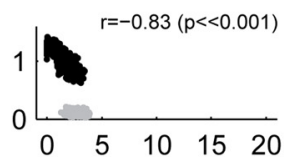

C3

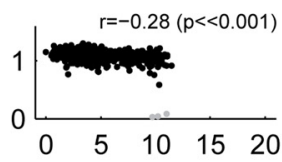

D3

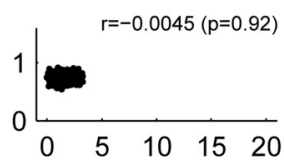

E3

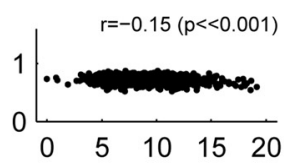

F3

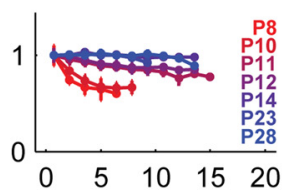

G3

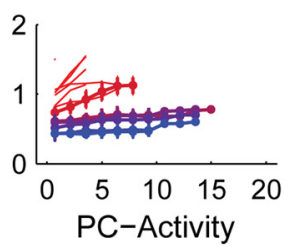

Figure 5. Postsynaptic AP amplitude and transmission delay are strongly influenced by the preceding activity in prehearing animals. $\boldsymbol{A}$, The preceding activity is quantified by the preceding interspike interval ( $\boldsymbol{A} \boldsymbol{1}$, left column, referring to presynaptic $\mathrm{AP}$, $\left.|S|_{P r e}\right)$, by a weighted average (exponential decay) of the preceding presynaptic ( $\boldsymbol{A} \mathbf{2}$, middle column, calyx-activity) or postsynaptic ( $A 3$, right column, PC-activity) AP train (for details see Materials and Methods). $A_{\text {Pre }}$ and $A_{\text {Post }}$ represent the amplitude of the presynaptic and postsynaptic $A P$, and $W_{i}$ is defined as $e^{\left(t_{i}-t_{0}\right) / \tau}$. Note, that high ISIs correspond to low activity values and vice versa. $\boldsymbol{B}$, In an exemplary unit of a prehearing animal (P8), $A_{\text {Post }}$ was strongly correlated with all three measures. Gray dots indicate failures of AP transmission. $C$, In an intermediate animal (P11) the dependence is weakened although still present with similar shapes. Gray dots indicate the few cases of failures of AP transmission. D, For a unit of a subadult animal (P23) with low spontaneous rate, no dependence of $A_{\text {Post }}$ on the preceding activity is observed. $E$, To compensate for the low spontaneous rate, the same analysis is shown for higher (stimulus-evoked) discharge rates for the same single-unit as shown in $\boldsymbol{D}$ (P23). Under these conditions, significant correlations are found for all three measures. Acoustic stimulation: $100 \mathrm{~ms}$ pure tones (100 ms interstimulus interval) at characteristic frequency and $60 \mathrm{~dB}$ sound pressure level (50 repetitions). $\boldsymbol{F}, \mathbf{G}$, On the population level the joint dependence of $A_{\text {Post }}(\boldsymbol{F})$ and also of transmission delay $(\boldsymbol{G})$ on age and level of preceding activity is observed in all three measures (different colors indicate different ages; see $\mathbf{G} 3$ legend) under spontaneous activity. While the strength of correlation is comparable for all three measures, the weighted activity measures (middle and right columns) tend to linearize the relationship. Only bins with 5 or more transmission events were evaluated. $A_{\text {Post }}$ was normalized in $F$ by dividing by the mean of the amplitude of the threelargestbins.

short-term depression, we found an increase in EPSP amplitude at shortest ISI $_{\text {Pre }}$, especially between P8 and P11, which might be indicative of synaptic facilitation, which has already been described for the calyx of Held (BarnesDavies and Forsythe, 1995; Borst et al., 1995; Müller et al., 2010). However, under spontaneous activity, only few events were preceded by such short ISI ${ }_{\mathrm{Pre}} \mathrm{s}$ (leading to the high variability in amplitude for the older ages), and hence short-term facilitation is suggested but cannot be clearly demonstrated by the current dataset.

\section{Discussion}

The present study details the development of many properties of synaptic transmission from juvenile to young adult stages under natural patterns of neuronal activity in vivo. We find that signal transmission at the mouse calyx of Held matures rapidly during the second postnatal week and acquires its adult-like properties around hearing onset, in parallel with the developmental changes in spontaneous discharge patterns. Further, many properties of synaptic transmission-especially the timing and amplitude of the postsynaptic AP — covary strongly during natural activity as a function of the temporally preceding activity in juvenile animals. While many of the present results have previously been obtained in vitro, our study resolves the uncertainty as to whether they translate to the situation in vivo where spontaneous activity patterns influence the state of the calyx of Held. Hence, the present study provides a rich source of in vivo data for previous and future in vitro studies with respect to both age and activity dependence of properties of synaptic transmission.

Further, we have introduced an advanced fitting approach which can aid extracellular in vivo studies to reach a new level of precision. Measuring properties of synaptic transmission is a complex task for closely spaced transmission events. The new method provides reliable estimates of many properties of synaptic transmission as indicated by the results and the match with previous in vitro data.

$$
\leftarrow
$$

Data of the same age group were averaged (only successful transmission events). For the transmission delays in $\mathbf{G}$, the absolute data were plotted and averaged for six postnatal ages (P10-P28), except for P8, where the transmission delays of individual single-units covered different ranges of activity levels. We therefore plotted individual P8 single-units to avoid a biased mean curve. The number of units per age is the same as indicated in Figure 4C4. Error bars correspond to $2 *$ SEM. 

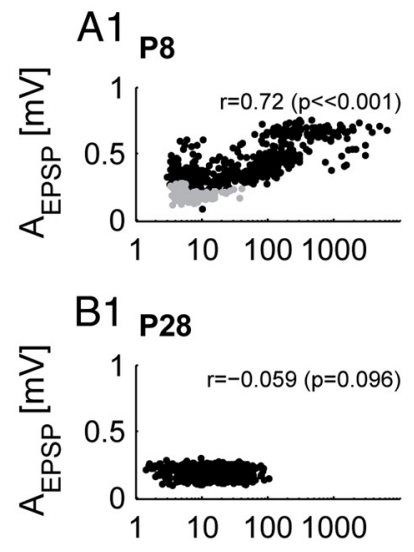

C1

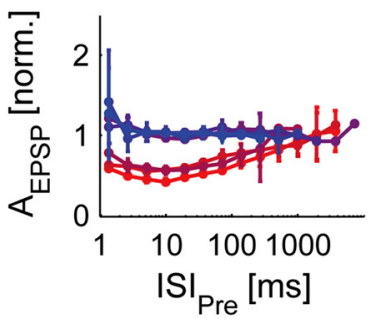

A2

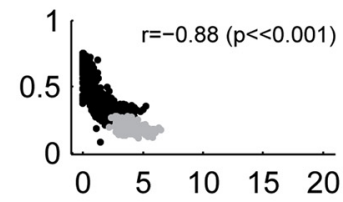

B2

A3

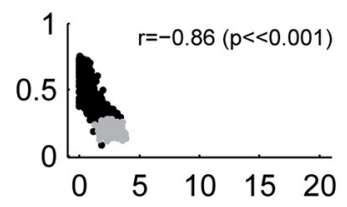

B3

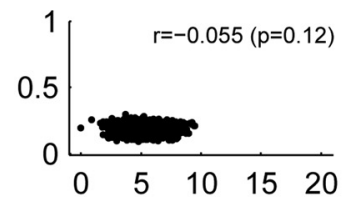

C2

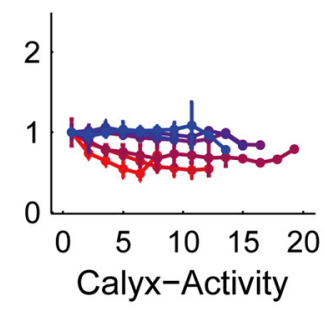

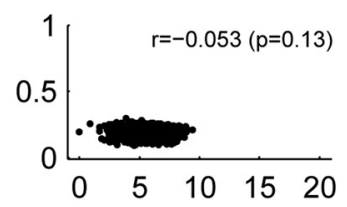

C3

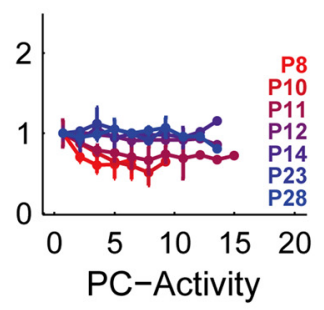

Figure 6. Size of EPSP is influenced by the preceding activity in prehearing animals. The preceding activity is quantified by the

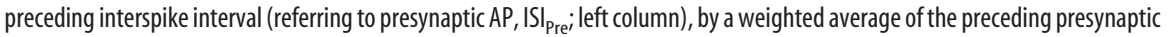
(middle column) or postsynaptic (right column) AP train (for details see Materials and Methods; Fig. 5A1-3). A, Example unit of a P8 animal. $A_{\text {EPSP }}$ was strongly dependent on all three measures of preceding activity. Gray dots indicate failures of AP transmission. $\boldsymbol{B}$, Example unit of a P28 animal. $A_{\text {EPSP }}$ did not change with different preceding activity levels. $\boldsymbol{C}$, Population data (different colors indicate different ages, legend see (3). In prehearing animals, $A_{\text {EPSP }}$ is reduced with decreasing $|S|_{\text {Pre, }}$ and increasing levels of preceding calyx- and PC-activity, respectively. Throughout development, $A_{\text {EPSP }}$ becomes uncorrelated to the preceding activity. Only bins with 5 or more transmission events were evaluated. $A_{\text {EPSP }}$ was normalized by dividing by the mean amplitude of the three largest bins. Data were averaged by postnatal day and error bars correspond to $2 *$ SEM. The number of units in each age group is the same as indicated in Figure 4C4. amine might reduce the frequency of AP failures in in vivo experiments by inhibition of NMDA receptors leading to reduced production of nitric oxide (Steinert et al., 2008). If this applies, the use of ketamine in the present study could have led to an underestimation of failure rates at young ages, when NMDA receptors contribute considerably to signal transmission. However, in our case no influence of ketamine on the reliability of signal transmission was apparent (data not shown). At the mature calyx, ketamine's influence on the reliability of transmission is expected to be strongly reduced since the levels of NMDA receptor expression are much lower (Futai et al., 2001; Joshi and Wang, 2002; Steinert et al., 2010).

However, with respect to the reliability of signal transmission at the mature calyx of Held, it is still discussed whether it acts as a close to failsafe relay (Mc Laughlin et al., 2008; Englitz et al., 2009) or not (Kopp-Scheinpflug et al., 2003, 2008; Lorteije et al., 2009). Several factors might explain these inconsistent results: (1) species differences, (2) differences in the specifics of anesthesia, (3) differences in the level of synaptic activation (spontaneous vs acoustically evoked activity) and consequently differences in the contribution of excitatory and inhibitory transmitters, (4) differences in the invasiveness of recording techniques, and (5) differences in analytical techniques.
Hence, it provides a useful tool for future pharmacological in vivo studies of synaptic transmission.

\section{Maturation of fast and reliable signal transmission}

As part of the sound localization pathway, a fast and reliable signal transmission at the calyx of Held is considered essential for accurate estimation of sound location. Our data indicate that these conditions are accomplished within $2 \mathrm{~d}$ after the onset of hearing. We found an increase in average transmission speed up to P14 accompanied by a shortening of presynaptic and postsynaptic signals and by a reduction of jitter of transmission speed. Underlying developmental changes in the kinetics of synaptic currents (Taschenberger and von Gersdorff, 2000) and in sodium and potassium conductance, affecting the AP dynamics, can account for the maturation of fast signal transmission (Ming and Wang, 2003; Leão et al., 2005; Nakamura and Takahashi, 2007). Furthermore, the calyx of Held's transmission reliability developed from frequently failing to failsafe by P14. While in the early second postnatal week (P8-P10) individual units revealed failure rates of up to $37 \%$, AP failures became rare at P12 $(<2 \%)$ and, finally, AP transmission appeared secure at P14. The high expression of NMDA receptors in the developing MNTB principal cell might contribute to the presence of failures up to P12. It has been suggested to induce a tonic depolarization inactivating sodium channels and reducing neuron excitability (Taschenberger and von Gersdorff, 2000; Futai et al., 2001; Joshi and Wang, 2002). A recent study suggested that the commonly used anesthetic ket-

\section{Development of activity-dependent plasticity}

We found clear developmental changes in activity-dependent plasticity of signal transmission at the calyx of Held. In prehearing animals, the amplitude of the EPSP was strongly depressed as a function of preceding activity (Fig. 6). Also AP failures were shown to mainly occur at high levels of preceding activity accompanied by small (and likely subthreshold) EPSPs and thus are likely caused by STD. In hearing animals, STD was not observed anymore (Lorteije et al., 2009) and, also, transmission failures were rare, suggesting that STD is of little relevance in hearing animals, at least during spontaneous activity. The underlying physiological conditions are the adult-like size of readily releasable vesicle pools, a sufficient exocytosis efficiency, and the reduced release probability compensated by tighter coupling of vesicles to calcium channels (Taschenberger and von Gersdorff, 2000; Futai et al., 2001; Iwasaki and Takahashi, 2001; Joshi and Wang, 2002; Taschenberger et al., 2002; Fedchyshyn and Wang, 2005; Yang and Wang, 2006; Wang et al., 2008; Kochubey et al., 2009; Leão and von Gersdorff, 2009). The transmission delay and the amplitude of the postsynaptic AP also underlie strong activity-dependent plasticity in prehearing mice and, to a lesser extent, also in hearing animals. One possible cause for the larger effects in prehearing mice is the immature state of sodium channel kinetics leading to long refractory periods and to reduced availability of sodium channels at high activity levels (Ming and Wang, 2003; Leão et al., 2005). However, when triggering higher activity levels by afferent fiber stimulation in vitro or by acoustic 
stimulation in vivo, significant activity-dependent changes in the transmission delay also occur at the mature calyx of Held (Fedchyshyn and Wang, 2007; Mc Laughlin et al., 2008; Tolnai et al., 2009). Thus, during high-frequency firing $(>200 \mathrm{~Hz})$, the retention of temporal precision of signal transmission might be limited even at the mature calyx of Held.

\section{Influence of ambient activity on the properties of signal transmission}

In hearing animals, most neurons are permanently active without long periods of quiescence. This continuous ambient activity was shown to severely influence synaptic transmission, especially the properties of STD. High spontaneous activity is expected to shift the STD into a tonic regime as demonstrated by Hermann et al. (2007). Under these conditions the mature calyx of Held enters a chronically depressed state, partly explaining the lack of STD in vivo (Lorteije et al., 2009; Borst, 2010), especially in units with high spontaneous rates. However, under slice conditions, STD remains significant at older ages, although only at high stimulation frequencies (Wang and Kaczmarek, 1998; Taschenberger and von Gersdorff, 2000; Lorteije et al., 2009).

Neurons in the developing afferent auditory pathway differ significantly from mature neurons with respect to their spontaneous spiking patterns. Before the onset of hearing, the immature cochlea is not susceptible to airborne sound. Still, the inner hair cells in the auditory sensory epithelium are not inactive, but are depolarized, triggering APs in auditory nerve fibers (Tritsch et al., 2007; Tritsch and Bergles, 2010). This prehearing, cochlear depolarization is mediated by ATP, which is released intermittently, causing bursts of discharges interrupted by silent or low-activity periods (Fig. 1) (Sonntag et al., 2009; Tritsch et al., 2010). Thus, the bursty stimulation typically applied in in vitro experiments resembles the natural activity patterns in prehearing auditory neurons quite well. In support of this observation, we found the properties of signal transmission during the second postnatal week in vivo to be in good agreement with earlier in vitro reports (Taschenberger and von Gersdorff, 2000; Futai et al., 2001; Joshi and Wang, 2002; for review, see von Gersdorff and Borst, 2002), although differences in synaptic physiology between in vivo and slice preparations are also present at these early stages; e.g., the occurrence of aberrant postsynaptic APs in acute brainstem slices (Futai et al., 2001) which were not found in the present in vivo study (as by Tritsch et al., 2010). In this regard, immediate metabolic and structural changes at the synapse induced by the loss of afferent activity during in vitro preparations may play an important role in synaptic homeostasis (Rubel et al., 1990; Rubel and Fritzsch, 2002). In conclusion, the ambient spontaneous activity and its age-specific temporal pattern should be recreated under slice conditions, especially when studying synaptic transmission in the critical period around hearing onset.

\section{Fast and reliable signal transmission in the context of sound localization}

In summary, we show that the development of signal transmission in vivo is completed around hearing onset, which is a pivotal precondition for the transmission of auditory information as soon as mice commence processing of acoustically evoked afferent activity. Even though the present results support the mature calyx synapse's proposed role as a relay with respect to reliability and timing, it must be considered that these results refer to spontaneous activity and might differ at higher rates, as has been found in vitro (Hermann et al., 2007; Steinert et al., 2008) but has been questioned in vivo (Mc Laughlin et al., 2008; Englitz et al.,
2009). With respect to its significance in signal processing, it will not be sufficient to clarify whether the calyx fails at all but, rather, under which conditions and to which degree transmission failures occur. This will be the determinant for its influence on the accuracy of sound localization. Consequently, one focus of future studies should be the influence of failures of transmission on sound localization and the conditions under which they occur.

\section{References}

Barnes-Davies M, Forsythe ID (1995) Pre- and postsynaptic glutamate receptors at a giant excitatory synapse in rat auditory brainstem slices. J Physiol 488:387-406.

Borst JG (2010) The low synaptic release probability in vivo. Trends Neurosci 33:259-266.

Borst JG, Helmchen F, Sakmann B (1995) Pre- and postsynaptic whole-cell recordings in the medial nucleus of the trapezoid body of the rat. J Physiol 489:825-840.

Englitz B, Tolnai S, Typlt M, Jost J, Rübsamen R (2009) Reliability of synaptic transmission at the synapses of Held in vivo under acoustic stimulation. PLoS One 4:e7014.

Erazo-Fischer E, Striessnig J, Taschenberger H (2007) The role of physiological afferent nerve activity during in vivo maturation of the calyx of Held synapse. J Neurosci 27:1725-1737.

Fedchyshyn MJ, Wang LY (2005) Developmental transformation of the release modality at the calyx of Held synapse. J Neurosci 25:4131-4140.

Fedchyshyn MJ, Wang LY (2007) Activity-dependent changes in temporal components of neurotransmission at the juvenile mouse calyx of Held synapse. J Physiol 581:581-602.

Friauf E, Lohmann C (1999) Development of auditory brainstem circuitry. Activity-dependent and activity-independent processes. Cell Tissue Res 297:187-195.

Futai K, Okada M, Matsuyama K, Takahashi T (2001) High-fidelity transmission acquired via a developmental decrease in NMDA receptor expression at an auditory synapse. J Neurosci 21:3342-3349.

Guinan JJ Jr, Li RY (1990) Signal processing in brainstem auditory neurons which receive giant endings (calyces of Held) in the medial nucleus of the trapezoid body of the cat. Hear Res 49:321-334.

Herbst JA, Gammeter S, Ferrero D, Hahnloser RH (2008) Spike sorting with hidden Markov models. J Neurosci Methods 174:126-134.

Hermann J, Pecka M, von Gersdorff H, Grothe B, Klug A (2007) Synaptic transmission at the calyx of Held under in vivo like activity levels. J Neurophysiol 98:807-820.

Iwasaki S, Takahashi T (2001) Developmental regulation of transmitter release at the calyx of Held in rat auditory brainstem. J Physiol 534:861-871.

Joshi I, Wang LY (2002) Developmental profiles of glutamate receptors and synaptic transmission at a single synapse in the mouse auditory brainstem. J Physiol 540:861-873.

Kochubey O, Han Y, Schneggenburger R (2009) Developmental regulation of the intracellular $\mathrm{Ca}^{2+}$ sensitivity of vesicle fusion and $\mathrm{Ca}^{2+}$-secretion coupling at the rat calyx of Held. J Physiol 587:3009-3023.

Kopp-Scheinpflug C, Lippe WR, Dörrscheidt GJ, Rübsamen R (2003) The medial nucleus of the trapezoid body in the gerbil is more than a relay: comparison of pre- and postsynaptic activity. J Assoc Res Otolaryngol $4: 1-23$.

Kopp-Scheinpflug C, Dehmel S, Tolnai S, Dietz B, Milenkovic I, Rübsamen R (2008) Glycine-mediated changes of onset reliability at a mammalian central synapse. Neuroscience 157:432-445.

Leão RM, von Gersdorff H (2009) Synaptic vesicle pool size, release probability and synaptic depression are sensitive to $\mathrm{Ca}^{2+}$ buffering capacity in the developing rat calyx of Held. Braz J Med Biol Res 42:94-104.

Leão RM, Kushmerick C, Pinaud R, Renden R, Li GL, Taschenberger H, Spirou G, Levinson SR, von Gersdorff H (2005) Presynaptic Na+ channels: locus, development, and recovery from inactivation at a high-fidelity synapse. J Neurosci 25:3724-3738.

Lorteije JA, Rusu SI, Kushmerick C, Borst JG (2009) Reliability and precision of the mouse calyx of Held synapse. J Neurosci 29:13770-13784.

McKay SM, Oleskevich S (2007) The role of spontaneous activity in development of the endbulb of Held synapse. Hear Res 230:53-63.

Mc Laughlin M, van der Heijden M, Joris PX (2008) How secure is in vivo synaptic transmission at the calyx of Held? J Neurosci 28:10206-10219. 
Ming G, Wang LY (2003) Properties of voltage-gated sodium channels in developing auditory neurons of the mouse in vitro. Chin Med Sci J 18:67-74.

Müller M, Goutman JD, Kochubey O, Schneggenburger R (2010) Interaction between facilitation and depression at a large CNS synapse reveals mechanisms of short-term plasticity. J Neurosci 30:2007-2016.

Nakamura Y, Takahashi T (2007) Developmental changes in potassium currents at the rat calyx of Held presynaptic terminal. J Physiol 581:1101-1112.

Rubel EW, Fritzsch B (2002) Auditory system development: primary auditory neurons and their targets. Annu Rev Neurosci 25:51-101.

Rubel EW, Hyson RL, Durham D (1990) Afferent regulation of neurons in the brain stem auditory system. J Neurobiol 21:169-196.

Schwarz G (1978) Estimating the dimension of a model. Ann Stat 6:461-464

Sonntag M, Englitz B, Kopp-Scheinpflug C, Rübsamen R (2009) Early postnatal development of spontaneous and acoustically evoked discharge activity of principal cells of the medial nucleus of the trapezoid body: an in vivo study in mice. J Neurosci 29:9510-9520.

Steinert JR, Kopp-Scheinpflug C, Baker C, Challiss RA, Mistry R, Haustein MD, Griffin SJ, Tong H, Graham BP, Forsythe ID (2008) Nitric oxide is a volume transmitter regulating postsynaptic excitability at a glutamatergic synapse. Neuron 60:642-656.

Steinert JR, Postlethwaite M, Jordan MD, Chernova T, Robinson SW, Forsythe ID (2010) NMDAR-mediated EPSCs are maintained and accelerate in time course during maturation of mouse and rat auditory brainstem in vitro. J Physiol 588:447-463.

Taschenberger H, von Gersdorff H (2000) Fine-tuning an auditory synapse for speed and fidelity: developmental changes in presynaptic waveform, EPSC kinetics, and synaptic plasticity. J Neurosci 20:9162-9173.

Taschenberger H, Leão RM, Rowland KC, Spirou GA, von Gersdorff $\mathrm{H}$ (2002) Optimizing synaptic architecture and efficiency for highfrequency transmission. Neuron 36:1127-1143.

Tolnai S, Englitz B, Scholbach J, Jost J, Rübsamen R (2009) Spike transmission delay at the calyx of Held in vivo: rate dependence, phenomenological modeling, and relevance for sound localization. J Neurophysiol 102:1206-1217.

Tritsch NX, Bergles DE (2010) Developmental regulation of spontaneous activity in the mammalian cochlea. J Neurosci 30:1539-1550.

Tritsch NX, Yi E, Gale JE, Glowatzki E, Bergles DE (2007) The origin of spontaneous activity in the developing auditory system. Nature 450:50-55.

Tritsch NX, Rodríguez-Contreras A, Crins TT, Wang HC, Borst JG, Bergles DE (2010) Calcium action potentials in hair cells pattern auditory neuron activity before hearing onset. Nat Neurosci 13:1050-1052.

von Gersdorff H, Borst JG (2002) Short-term plasticity at the calyx of held. Nat Rev Neurosci 3:53-64.

Wang LY, Kaczmarek LK (1998) High-frequency firing helps replenish the readily releasable pool of synaptic vesicles. Nature 394:384-388.

Wang LY, Neher E, Taschenberger M (2008) Synaptic vesicles in mature calyx of Held synapses sense higher nanotomain calcium concentrations during action potential-evoked glutamate release. J Neurosci 28:14450 14458.

Yang YM, Wang LY (2006) Amplitude and kinetics of action potentialevoked $\mathrm{Ca}^{2+}$ current and its efficacy in triggering transmitter release at the developing calyx of held synapse. J Neurosci 26:5698-5708. 